\title{
Immunculus as an Approach for the Evaluation of Multiple Sclerosis: A Case Report
}

\author{
Paria Jorbozedar ${ }^{1}$, Mir Hadi Jazayeri*1,2, Mohamad Ebrahimi ${ }^{3}$ and Amir Avan ${ }^{4}$ \\ ${ }^{1}$ Department of Immunology, Iran \\ ${ }^{2}$ Immunology Research Center, Iran
}

${ }^{3}$ Department of Immunogenetic, Iran

${ }^{4}$ Metabolic syndrome Research center, Iran

Received: 眥: December 04, 2018; Published: 制: December 18, 2018

*Corresponding author: Mir Hadi Jazayeri, Department of Immunology, Tehran, Iran

\begin{abstract}
Fingolimod is the first agent used for the treatment of relapsing-remitting multiple sclerosis. Here we report a case, who was diagnosed with multiple sclerosis, after receiving fingolimod treatment feels well except mild tingling in his hands and feet. Repeated cell counts using flowcytometery, showed that his T cell count using CD3 marker was progressively decreased $(138 / \mu \mathrm{L}$, compared to normal range of $955-2860 / \mu \mathrm{L})$. Also his NK cell count using CD16 marker, showed 55.77 percent cells in his body, which was much more higher than normal range, suggesting the good condition of our patient at the time being. The CD34 marker known as the marker of hematopoietic progenitor cells was three folds higher than normal range. Interestingly ELI-viscero 24 test revealed autoreactivity against platelet and insulin surpassed, which was a concern for the patient about developing autoimmune disorders. Although our patient is now feels well, but his condition could be complicated in early future by many risk factors, in spite of dramatic drop in his T cells, and continuing to receive fingolimod may not be beneficial for him and other patients with this condition. Moreover it is suggestive that the present of high amount of NK cell might play a protective role and might serve to repair the lesions of CNS.
\end{abstract}

Keywords: Multiple Sclerosis; Immunculus; Natural Antibody Profile; Fingolimod

Abbreviations: MS: Multiple Sclerosis; CNS: Central Nervous System; HSPCs: Hematopoietic Stem/Progenitor cells; Th: T helper

\section{Introduction}

Multiple Sclerosis (MS) is the most common demyelinating chronic inflammatory disease of central nervous system (CNS) with incidence of 90 in 100000 populations [1,2]. It has been reported that MS is a multifactorial disease medicated by the interaction of genetic and environmental factors [3]. Several studies have carried out to underlie the etiopathogenesis of MS, although many important questions are still remained to be elucidated on the role of immune system with development/progression of the disease [2]. There is growing body of evidence showing MS as an autoimmunity disorder with the role of cytotoxic T cells and Th1/ Th17 cells [4]. Several approaches have developed for modulating cellular immunity including corticosteroids, interferons, glatiramer acetate, natalizumab, mitoxantrone, alemtuzumab and fingolimod. Fingolimod is approved for treating MS, via trapping T lymphocytes in lymph nodes through ligation with sphingosine-1-phosphate receptor, leading to internalization of the receptor and preventing $\mathrm{T}$ cells from recruitment to CNS [5]. Moreover it has been suggested that this agent is able to inhibit cytotoxic $\mathrm{T}$ cells and thereby affecting arachidonic acid pathway [6], although several side effects have also reported such as lymphocytopenia leading to infections and hypertension, bradycardia, macular edema, fatigue, and headache [7]. Against this background, ELI-viscero 24 test analyses 24 antibodies against auto antigens [8].

\section{Methods}

\section{Case Presentation}

A 32 year old male Iranian (fars) patient living in Tehran, with severe backache presented to familiar physician in 2012. He had no history of any immune related disorders or chronic diseases and no long term medication prescription. After physical examination, based on his symptom and history, he was misdiagnosed for a common backache and prescribed with a course of non-steroidal anti-inflammatory drugs and hot tub to relieve his pain, after which the problem was completely resolved for two years without any 
symptoms. In 2014 he represented to physician again suffering from severe backache, as well as pain in left leg, which caused difficulty in walking. These symptoms lasted more than a week and did not respond completely to medication. According to MRI, he was diagnosed with MS and then prescribed one week treatment with methyl prednisolone pulse, after which he was feeling well (Figure
1). He had not had any symptoms for about one year. In 2015 the disease manifested again and this time he was prescribed with Interferon- $\beta$ and after a short period with fingolimod. In the course of fingolimod treatment he feels well except tingling in his hands and feet, and pain in left leg, and also general feeling of weakness and nervousness.

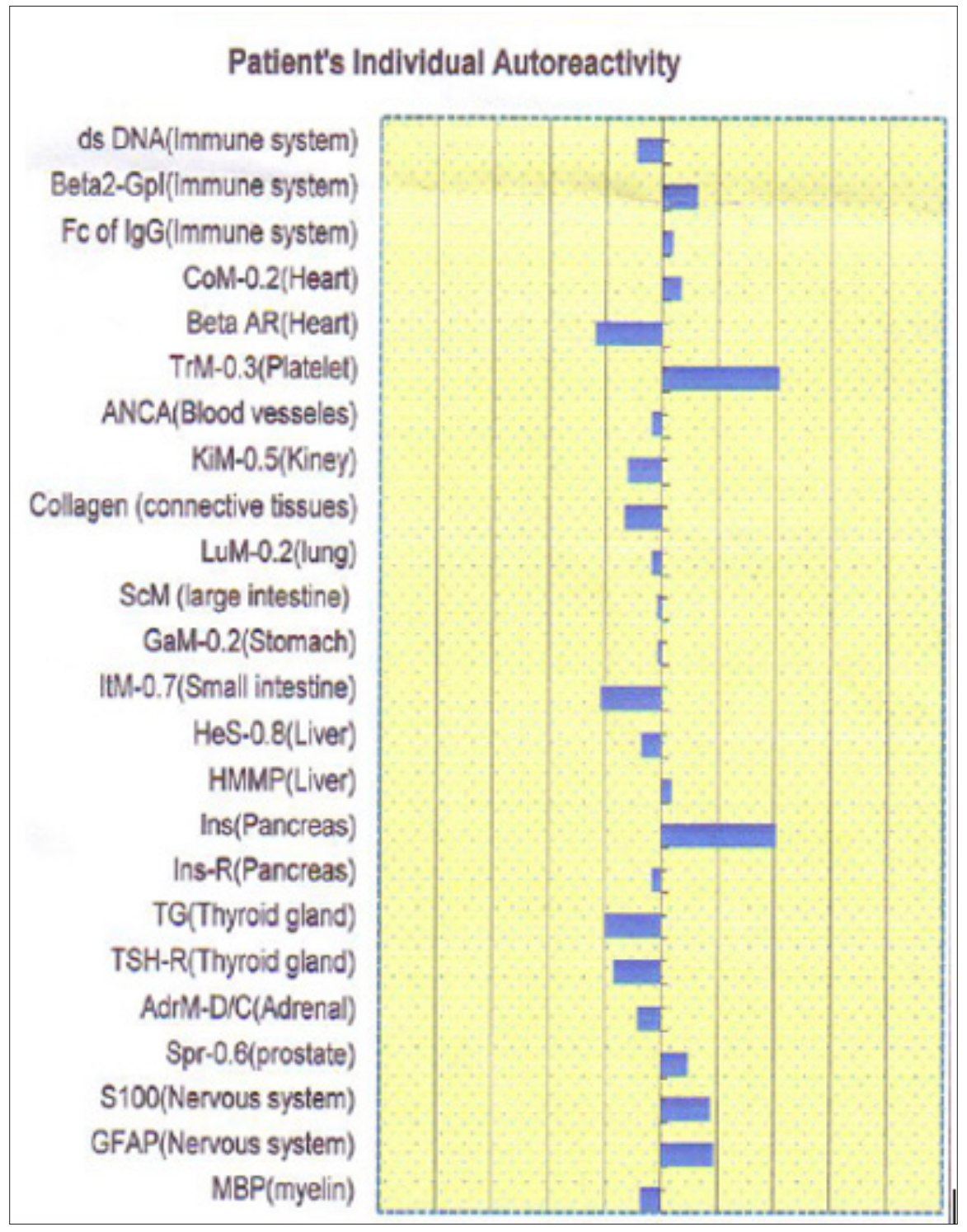

Figure 1: Patient's individual auto-reactivity.

For the reason of uncomfortable tingling, he was referred to Farda Clinic. After medical consultation and performing routine laboratory tests, including complete blood count and flowcytometery, it was revealed that he is lymphopenic with lymphocyte absolute count of 510/ $\mu \mathrm{L}$, compared to normal range of 1530-3700. His $\mathrm{T}$ cell absolute count was as low as $138 / \mu \mathrm{L}$ and was dropping in subsequent tests. Also, his NK cell percent using CD16 marker was significantly higher than normal range of 5-19 percent and was 55.77 percent. Another noticeable finding in flowcytometry tests was that the CD34 marker in his blood was higher than normal range (3.33 compared with normal range of less than 1 percent in lymphocytes). Besides, we carried out an ELI-viscero 24 test for him and it was revealed that his autoreactivity against platelet and insulin falls in high regions with values 41.3 and 40.9 respectively. All other cell counts including B cell count using CD20 marker were normal. In examination of pathogenic agents using immunologic tests, high Immunoglobulin G against Cytomegalovirus, herpes simplex virus, and Epstein barr virus was detected (Figure 2). 


\begin{tabular}{|c|c|c|c|}
\hline Antigen (Target Organ/Tissue) & $\begin{array}{l}\text { Ratio } \\
\text { Value }\end{array}$ & Antigen & $\begin{array}{l}\text { Normalized } \\
\text { Autoreactivity }\end{array}$ \\
\hline dsDNA (Immune system) & -54.1 & ds DNA(Immune system) & $\begin{array}{r}-9.4 \\
\end{array}$ \\
\hline beta2-GPI (Immune system) & -32.6 & Beta2-Gpl(Immune system) & 12.2 \\
\hline Fc fragment of IgG (Immune system) & -41.0 & Fc of $\lg G$ (Immune system) & 3.7 \\
\hline CoM-0.2Membrane Ag of myocardial cells(Heart) & 37.9 & CoM-0.2(Heart) & 6.8 \\
\hline betaAR Beta Adrenoreceptor(Heart) & -68.5 & Beta AR(Heart) & -23.8 \\
\hline TrM-0.3Membrane Ag of platelets & -3.4 & TrM-0.3(Platelet) & 41.3 \\
\hline ANCA Anionic Ag of endotholium(blood vesseles) & -48.6 & ANCA(Blood vesseles) & -3.9 \\
\hline KiM-0.5Membrane Ag of Kidney & -57.0 & \begin{tabular}{|c|} 
KiM-0.5(Kiney) \\
\end{tabular} & -12.3 \\
\hline Collagen Main structural protein of connective tissues & -57.9 & Collagen (connective tissues) & -13.2 \\
\hline LuM-0.2Membrane $\mathrm{Ag}$ of lungs & -48.5 & \begin{tabular}{|c|} 
LuM-0.2(lung) \\
\end{tabular} & -3.8 \\
\hline ScM antigen of S. Cerevisiae & -46.4 & ScM (large intestine) & -1.7 \\
\hline GaM-0.2Membrane Ag of Stomach & -46.2 & GaM-0.2(Stomach) & -1.5 \\
\hline ItM-0.7Membrane Ag of Small Intestine & -66.5 & ItM-0.7(Small intestine) & -21.8 \\
\hline HeS- 0.8 Cytoplasmic Ag of Liver & -51.9 & HeS-0.8(Liver) & -7.2 \\
\hline HMMP Ag of Liver mytochondria & -41.0 & HMMP(Liver) & 3.7 \\
\hline Insulin (Pancreas) & -3.8 & Ins(Pancreas) & 40.9 \\
\hline InS-R (Insulin Reseptors) & -48.2 & Ins-R(Pancreas) & -3.5 \\
\hline TG Thyroglobulin (Thyroid gland) & -64.6 & TG(Thyroid gland) & -19.9 \\
\hline TSH-R (Thyroid gland) & -61.7 & TSH-R(Thyroid gland) & -17.0 \\
\hline AdrM-D/C Membrane Ag of Adrenal gland & -53.2 & AdrM-D/C(Adrenal) & -8.5 \\
\hline Spr-0.6MEmbranous Ag of Prostate gland & -35.1 & Spr-0.6(prostate) & 9.6 \\
\hline S100 (Nervous system) & -27.1 & S100(Nervous system) & 17.6 \\
\hline GFAP Protein of Intercurrent Astrocytes filaments (Ner) & -25.8 & GFAP(Nervous system) & 18.9 \\
\hline MBP Myelin Basic Protein (myelln) & -51.8 & MBP(myelin) & -7.1 \\
\hline
\end{tabular}

Figure 1: Patient's individual auto-reactivity.

\section{Discussion}

MS is a complex and heterogenic disease. Some people have few and mild symptoms, while others might have severe symptoms, with different effectiveness of treatment. One of the new technologies in assessment of healthy status is immunculus or immunological homonculus [8]. It is now widely accepted that auto-Abs are permanently and obligatorily produced in all healthy individuals. According to the immunochemical homeostasis (immunculus) concept, rates of production of natural auto-Ab are regulated by quantity changes of respective antigens via a feedback principle. The human B-1 cell are responsible for the secretion of natural autoAbes with no stimulation from the early stages of embryogenesis, which are changed during the life [9]. The rates of production, secretion and/or release of any cytoplasmic, membranous, or nuclear antigen into the intercellular space are nearly equivalent or differ insignificantly in all healthy individuals; therefore, serum levels of respective auto-Abs should also demonstrate only slight individual variability. The natural antibodies profile changes dramatically in progression of diseases. Many chronic disorders, are directly associated with either abnormally elevated apoptosis or cell necrosis in the given tissues and subsequently deviations in the normal pattern of antigens.

In turn, a stable increase in the extracellular concentration of any endogenous antigen is inevitably accompanied by deviations in the concentrations of cognate auto-Abs according to Immunculus rule. The network of natural antibodies and their dynamically changes named Immunculus. Regulatory auto-antibodies and lymphocytes dynamically participate in self-maintenance, self-reparation, self- optimization, and maintenance of body under the constant changes of the environment. One of the most important biomolecules used by organisms is natural auto-Abs of the IgG and IgM class. The most peculiar feature of auto-Abs is their permanent presence in nearly every compartment of the body. The concentration of total IgG and IgM varies at different age intervals [10]. Therefore, quantitative evaluation of auto-Abs directed against specific sets of antigens of the given organs in a blood sample, regardless of origin, may provide equally valuable information about the state of specialized cells and tissue (normal/abnormal) and allowing us to monitor the healthy state of organ. In other words, the immunological "internal image "as holistic approaches presents a multi-dimensional "molecular model" of the body. The patient under treatment of fingolimod has complained of tingling in his back, mild pain in left leg, and also general feeling of weakness and nervousness. The results of Eliviscero-test with 24 different bio-markers showed a weak increased level of Beta2-glygoprotein I (12.2), severe increased level of Trm ( membrane antigen of platelet) (41.3), severe increased level of $\mathrm{Ab}$ against insulin (40.9) and also increased level of Ab against S100 (17.6) and GFAP (18.9).

The level of other Abs, including Ab against MBP, was in normal range. Meanwhile, the index of MIR (mean immune reactivity) was -44.70 , indicating polyclonal immune suppression (immune deficiency). These data gave evidence of transitory anti-phospholipide syndrome, changed platelet's functions and coagulopathy, chronic pancreatitis and reactive gliosis. In the last year, reactive gliosis has been used as a marker of pathology in multiple sclerosis [11]. S100 $\beta$ is also used as a complement to GFAP 
as a marker of neurotoxicity [12]. our previous results showed the mean immune reactivity of serum antibodies with 24 human autoantigens increased with advancing age, which could be because of the increase in the level of pathological antibodies in elderly [13]. Lymphocytopenia and low number of his T cells may pose him at high risk of infections and cancer; as the immunologic test results for CMV, HSV and EBV are far more than positive and indicative of a low cellular immunity activity. Furthermore, it has been shown that NAAbs regulate immune system and a large number of NAbs have a key role in tissue homeostasis. Immunosurveillance mechanism has a role in cancer prevention and the presence of NAbs in normal range/activity in healthy individuals is essential for body to maintain hemostasis [11].

Lowering $\mathrm{T}$ cells is one of effect of fingolimod as its mechanisms of action but surpassing the minimal count necessary for maintaining immunological function of body has no long-term benefit for patient [12]; and in case of our patient which his $\mathrm{T}$ cell count was progressively decreased in each subsequent test, the situation gets worsen and worsen. The impact of fingolimod is different on various immune cell populations, thus we believe that the high number of NK cells in our patient is due to fingolimod [13]. Today we were unable to find the exact effect of fingolimod on NK cells, some articles say fingolimod prescription leads to increment of NK cells, while others say it lowers the number of NK cells [14]. There are controversial data about NK cells role in MS, and two opposite roles has been proposed for them by researchers; one destructive pathogenic role through priming T helper (Th) 1 and Th17 and direct cell cytotoxicity through perforin secretion, and one protective immunoregulatory role via engaging their CD95 with CD95 ligand on pathogenic T cells which results in their apoptosis [15]. NK cells are classified in two main subgroups, including CD3CD16+CD56dim which predominates in Blood circulation consisting about 90 percent of circulating NK cells and has cytotoxic activity, and CD3-CD16+CD56bright which is the smaller population (about 10 percent in circulation) expressing high levels of cytokines and low levels of perforin.

The CD56bright subpopulation is considered immature and is mainly found in secondary lymph nodes; recently it has been shown that they are also found in cerebrospinal fluid. Also, NK cells can be classified based on expressing CD 95 on their surface [16]. As mentioned, it was shown that the CD34 marker was high in blood circulation of the patient; this marker is known as the marker of a group of cells called hematopoietic stem/progenitor cells (HSPCs), we don't know the exact phenotype of these cells and the functions they exert on the body, but there are accumulative evidence that these cells can contribute to the repair of damages of the body organs $[17,18]$. As the overall condition of our patient is good, it is suggestive that these cells play a protective role and maybe serve to repair the lesions of CNS. May be the rise of these cells is due to the effect of fingolimod or is due to the lesions of MS, as these cells have tropism toward injuries. In a treatment regimen using natalizumab for regression of MS disease, the HSPCs increase in circulation and this increment is a sign of respond to the treatment [19].Alternative medicines with lower side effects and developing new strategies for treatment of MS and other immune disorders with more targeted therapies which deplete only autoreactive $\mathrm{T}$ cells are much more recommended and may help patients [20-22].

\section{Highlights}

a) Natural auto-antibody in physiological state is considered as an Immunculus.

b) Fingolimod is the first agent used for the treatment of relapsing-remitting multiple sclerosis..

c) ELI-viscero 24 test revealed auto-reactivity against platelet and insulin might be a predicting factor for autoimmune disorders.

\section{References}

1. Dilokthornsakul P, Valuck RJ, Nair KV, Corboy JR, Allen RR, et al. (2016) Multiple sclerosis prevalence in the United States commercially insured population. Neurology 86(11): 1014-1021.

2. Grigoriadis N, van Pesch V, Paradig MSG (2015) A basic overview of multiple sclerosis immunopathology. Eur J Neurol 2: 3-13.

3. Kutzelnigg A, Lassmann H (2014) Pathology of multiple sclerosis and related inflammatory demyelinating diseases. Handb Clin Neurol 122: 15-58.

4. Legroux L, Arbour N (2015) Multiple Sclerosis and T Lymphocytes: An Entangled Story. J Neuroimmune Pharmacol 10(4): 528-546.

5. Al Khamis FA (2016) The use of immune modulating drugs for the treatment of multiple sclerosis. Neurosciences (Riyadh) 21(1): 4-9.

6. Ntranos A, Hall O, Robinson DP, Grishkan IV, Schott JT, et al. (2014) FTY720 impairs CD8 T-cell function independently of the sphingosine1-phosphate pathway. Journal of neuroimmunology 270(1-2): 13-21.

7. Russo M, Guarneri C, Mazzon E, Sessa E, Bramanti P, et al. (2015) Fingolimod-Associated Peripheral Vascular Adverse Effects. Mayo Clin Proc 90(10): 1424-1427.

8. Poletaev AB, Churilov LP, Stroev YI, Agapov MM (2012) Immunophysiology versus immunopathology: Natural autoimmunity in human health and disease. Pathophysiology 19(3): 221-231.

9. Jazayeri MH, Pourfathollah AA, Jafari ME, Rasaee MJ, Dargahi ZV (2013) The association between human B-1 cell frequency and aging: From cord blood to the elderly. Biomedicine \& Aging Pathology 3(1): 20-22.

10. Jazayeri MH, Pourfathollah AA, Rasaee MJ, Porpak Z, Jafari ME (2013) The concentration of total serum IgG and IgM in sera of healthy individuals varies at different age intervals. Biomedicine \& Aging Pathology 3(4): 241-245.

11. Ebrahimnezhad S, Jazayeri M, Hassanian SM, Avan A (2017) Current status and prospective regarding the therapeutic potential of natural autoantibodies in cancer therapy. J Cell Physiol 232(10): 2649-2652.

12. Eng LF (1985) Glial fibrillary acidic protein (GFAP): the major protein of glial intermediate filaments in differentiated astrocytes. Journal of neuroimmunology 8(4-6): 203-214.

13. Korfias S, Stranjalis G, Papadimitriou A, Psachoulia C, Daskalakis G, et al. (2006) Serum S-100B protein as a biochemical marker of brain injury: a review of current concepts. Curr Med Chem 13(30): 3719-3731.

14. Jazayeri MH, Pourfathollah AA, Rasaee MJ, Farhadi M, Zarei N, et al. (2013) The reactivity of human serum natural autoantibodies with certain autoantigens increases along with aging. Biomedicine \& Aging Pathology 3(3): 115-118. 
15. Cervera C (2012) Infections and fingolimod. Rev Neurol 55(4): 227-237.

16. Kowarik MC, Pellkofer HL, Cepok S, Korn T, Kumpfel T, et al. (2011) Differential effects of fingolimod (FTY720) on immune cells in the CSF and blood of patients with MS. Neurology 76(14): 1214-1221.

17. Johnson TA, Evans BL, Durafourt BA, Blain M, Lapierre Y, et al. (2011) Reduction of the peripheral blood CD56(bright) NK lymphocyte subset in FTY720-treated multiple sclerosis patients. J Immunol 187(1): 570579.

18. Chanvillard C, Jacolik RF, Infante Duarte C, Nayak RC (2013) The role of natural killer cells in multiple sclerosis and their therapeutic implications. Front Immunol 4: 63.

19. Maghazachi AA (2013) On the role of natural killer cells in neurodegenerative diseases. Toxins (Basel) 5(2): 363-375.

ISSN: 2574-1241

DOI: 10.26717/BJSTR.2018.12.002237

Mir Hadi Jazayeri. Biomed J Sci \& Tech Res

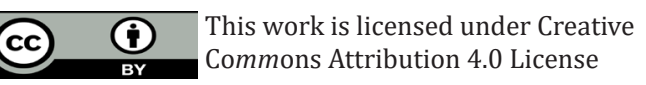

Submission Link: https://biomedres.us/submit-manuscript.php
20. Davoust N, Vuaillat C, Cavillon G, Domenget C, Hatterer E, et al. (2006) Bone marrow $\mathrm{CD} 34+\mathrm{B} 220+$ progenitors target the inflamed brain and display in vitro differentiation potential toward microglia. FASEB J 20(12): 2081-2092.

21. Bonig H, Wundes A, Chang KH, Lucas S, Papayannopoulou T (2008) Increased numbers of circulating hematopoietic stem/progenitor cells are chronically maintained in patients treated with the CD49d blocking antibody natalizumab. Blood 111(7): 3439-3441.

22. Mattoscio M, Nicholas R, Sormani MP, Malik O, Lee JS, et al. (2015) Hematopoietic mobilization: Potential biomarker of response to natalizumab in multiple sclerosis. Neurology 84(14): 1473-1482.

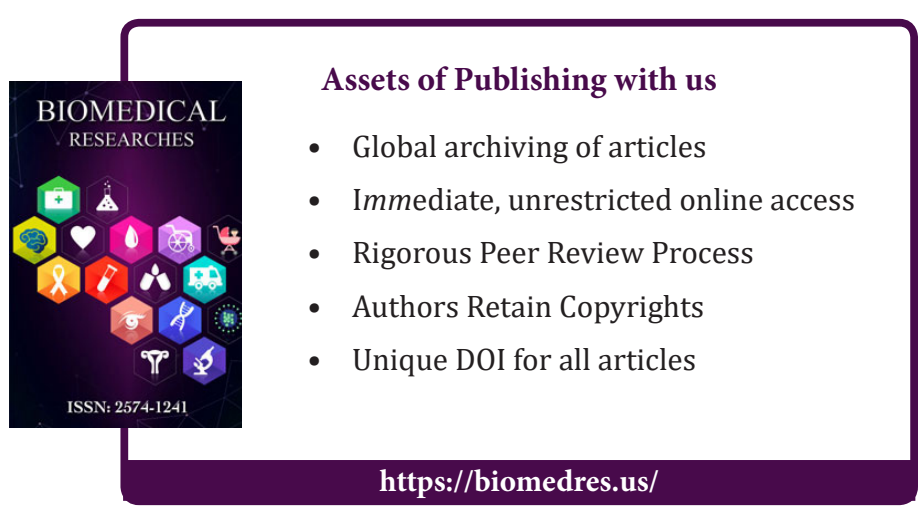

